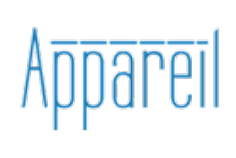

Appareil

$1 \mid 2008$

Le milieu des appareils

\title{
Le téléphone : milieu de souffle
}

\section{Olivier Leplatre}

\section{(2) OpenEdition}

\section{Journals}

Édition électronique

URL : http://journals.openedition.org/appareil/121

DOI : 10.4000/appareil. 121

ISSN : 2101-0714

\section{Éditeur}

MSH Paris Nord

\section{Référence électronique}

Olivier Leplatre, «Le téléphone : milieu de souffle », Appareil [En ligne], 1 | 2008, mis en ligne le 09 février 2008, consulté le 30 juillet 2020. URL : http://journals.openedition.org/appareil/121 ; DOI : https://doi.org/10.4000/appareil.121

Ce document a été généré automatiquement le 30 juillet 2020.

\section{(c) (i) (2) $\Theta$}

Appareil est mis à disposition selon les termes de la Licence Creative Commons Attribution - Pas d'Utilisation Commerciale - Pas de Modification 4.0 International. 


\title{
Le téléphone : milieu de souffle
}

\author{
Olivier Leplatre
}

\section{NOTE DE L'AUTEUR}

On aura reconnu dès son titre où cette étude a trouvé son inspiration. Les travaux de Georges Didi-Huberman viendront tout au long de l'analyse faire respirer la pensée. Parmi eux : L'Homme qui marchait dans la couleur, Paris, Minuit, 2001 et Gestes d'air et de pierre. Corps, parole, souffle, image, Paris, Minuit, 2005.

\section{«Comme le voyageur qui navigue entre les îles de l'Archipel voit la buée lumineuse se lever sur le soir, et découvre peu à peu la ligne du rivage, je commence à apercevoir le profil de ma mort » \\ M. Yourcenar, Mémoires d'Hadrien}

1 Nous le savons d'un douloureux savoir : nos morts, les morts ont en nous à peine une voix, à peine un visage. Nous reste comme le substrat de leur respiration, perdure en nous quelque chose comme leur atmosphère. Ce qui d'eux rayonne, résonne, cette aura émanant du plus loin de leur absence revient nous toucher : elle nous pénètre, nous trouble, elle nous émeut de toute notre affection. Apparaissant dans leur nimbe atmosphérique, dans leur arôme de fantôme, les morts réclament, comme l'écrit Hubertus Tellenbach, le «flairement ", l'« inspiration » de notre mémoire ${ }^{1}:$ non de notre mémoire mémorative qui s'efforce en vain de se souvenir et de représenter, de tracer la forme du visage et de distinguer la voix, mais de notre mémoire réminiscente qui sait recevoir, au bord de l'image et de la voix, le souffle de l'absence, son « souffle indistinct ", selon l'envoûtante expression de Pierre Fédida²

2 C'est dans le mouvement de leur haleine que les morts s'adressent à nous et que nous pouvons les rêver si, à en croire Héraclite, l'homme dormant dans la nuit «touche au mort les yeux éteints" (fragment 26). L'émanation d'air des morts, ombre de leurs visages, silence de leurs voix, les «lève » - encore un mot de Pierre Fédida -, comme l'on dit d'un vent qu'il se lève. Aussi l'aire bouleversante de la réminiscence est-elle 
avant tout un milieu en mouvement, recevant des ondes de souffle. L'absence s'y fait sentir moins comme une empreinte, "encore trop soumise à du corps représentable ", que comme un contact, qui fait « revenir une présence sans la moindre possibilité de la représenter ${ }^{3}$ ». Notre sensibilité aux morts, à cette matière, à ce matériau esthésique mobile qui les remue encore et nous remue, est inséparable d'un appel et même de l'échange d'un appel, de deux appels qui s'appellent et se répondent : deux appels d'air, celui de leur survivance dans notre mémoire et de notre mémoire dans leur survivance.

Le téléphone a vocation à en être l'instrument sensible, à créer, dans son milieu d'espace et de temps, d'espace et de temps qui vont et viennent, le contact avec les morts, et donc, plus largement encore, le contact avec la survivance qui souffle dans la vie, depuis l'influence en nous de la mort. Formidable appareil de souffle que le téléphone ! Boîte noire, amplificateur atmosphérique, c'est un appareil d'absence, de remise de toute présence à sa singularité et à son étrangeté d'absence. Quand nous appelons ou quand nous sommes appelés, nous ne communiquons pas d'abord des mots, nous entrons, bien au-delà, dans l'abîme de l'appel. Nous saisit l'enjeu d'une demande extrême qui déchire l'écoute et nous convoque au plus loin de nous-mêmes. Au téléphone, avant toute parole, et tout visage étant absenté, nous ouvrons grand la possibilité du souffle par quoi nous nous donnons dans notre atmosphère, comme dans notre dernier souffle de vivant, ou notre respiration de fantôme. Et nous entendons la palpitation d'air de l'autre: son "souffle indistinct». En tout appel, se signifient l'effacement et l'amuïssement de l'être ; en tout appel, un au-delà se porte infiniment qui éclipse notre présence et la fait trembler dans l'haleine de notre voix, où déjà s'est pulvérisé notre visage. Ainsi, à travers le téléphone, de bouche à oreille, dans le geste de pure sensation qui est celui de la confidence et du murmure, nous partageons entre nous des souffles, nos souffles d'absence, les souffles de nos absents.

On pourrait prêter l'oreille ici à l'écho des mots, au commun vent acoustique qui quelquefois les rapproche, emportant avec eux le sens. On entendrait alors cette rumeur entre notre "Allô!» phatique, d'où se lancent nos messages, et ce beau verbe grec homérique " aîô » qui signifie aussi bien «j'entends », "je vois » que «j'exhale».

C'est tout juste une parole, le «allô ! " inaugural, à l'orée des mots, frémissant de souffle, quelque chose d'élégiaque aussi, l'esquisse d'une plainte : un « hélas! ${ }^{4}$.

\section{La vocation de la voix. Proust}

« un morceau d'air formé par un fil » André du Bouchet

Quand le narrateur de La Recherche du temps perdu, après un silence de quelques instants, entend la voix de sa grand-mère pour la première fois au téléphone, il lui arrive un événement inoubliable : cette voix familière, si souvent venue à l'oreille, mais toujours accompagnée du visage, se décante là, au téléphone, par l'entremise de son filtre sensible. Elle se dépose dans l'immanence. Elle est d'une douceur extrême, douceur de toutes les douceurs, et d'une tristesse mêlée. Pour la première fois, le narrateur entend le souffle de mort de sa grand-mère comme l'épiphanie de sa plus dense vérité :

Et aussitôt que notre appel a retenti, dans la nuit pleine d'apparitions sur laquelle nos oreilles s'ouvrent seules, un bruit léger - un bruit abstrait - celui de la distance supprimée - et la voix de l'être cher s'adresse à nous.

C'est lui, c'est sa voix qui nous parle, qui est là. Mais comme elle est loin! Que de 
fois je n'ai pu l'écouter sans angoisse, comme si devant cette impossibilité de voir, avant de longues heures de voyage, celle dont la voix était si près de mon oreille, je sentais mieux ce qu'il y a de décevant dans l'apparence du rapprochement le plus doux, et à quelle distance nous pouvons être des personnes aimées au moment où il semble que nous n'aurions qu'à étendre la main pour les retenir. Présence réelle que cette voix si proche - dans la séparation effective! Mais anticipation aussi d'une séparation éternelle! Bien souvent, écoutant de la sorte, sans voir celle qui me parlait de si loin, il m'a semblé que cette voix clamait des profondeurs d'où l'on ne remonte pas, et j'ai connu l'anxiété qui allait m'étreindre un jour, quand une voix reviendrait ainsi (seule et ne tenant plus à un corps que je ne devais jamais revoir) murmurer à mon oreille des paroles que j'aurais voulu embrasser au passage sur des lèvres à jamais en poussière.

Ce jour-là, hélas, à Doncières, le miracle n'eut pas lieu. Quand j'arrivai au bureau de poste, ma grand'mère m'avait déjà demandé ; j'entrai dans la cabine, la ligne était prise, quelqu'un causait qui ne savait pas sans doute qu'il n'y avait personne pour lui répondre car, quand j'amenai à moi le récepteur, ce morceau de bois se mit à parler comme Polichinelle; je le fis taire, ainsi qu'au guignol, en le remettant à sa place, mais, comme Polichinelle, dès que je le ramenais près de moi, il recommençait son bavardage. Je finis, en désespoir de cause, en raccrochant définitivement le récepteur, par étouffer les convulsions de ce tronçon sonore qui jacassa jusqu'à la dernière seconde et j'allai chercher l'employé qui me dit d'attendre un instant ; puis je parlai, et après quelques instants de silence, tout d'un coup j'entendis cette voix que je croyais à tort connaître si bien, car jusque-là, chaque fois que ma grand'mère avait causé avec moi, ce qu'elle me disait, je l'avais toujours suivi sur la partition ouverte de son visage où les yeux tenaient beaucoup de place ; mais sa voix elle-même, je l'écoutais aujourd'hui pour la première fois. Et parce que cette voix m'apparaissait changée dans ses proportions dès l'instant qu'elle était un tout, et m'arrivait ainsi seule et sans l'accompagnement des traits de la figure, je découvris combien cette voix était douce; peut-être d'ailleurs ne l'avait-elle jamais été à ce point, car ma grand'mère, me sentant loin et malheureux, croyait pouvoir s'abandonner à l'effusion d'une tendresse que, par "principes " d'éducatrice, elle contenait et cachait d'habitude. Elle était douce, mais aussi comme elle était triste, d'abord à cause de sa douceur même presque décantée, plus que peu de voix humaines ont jamais dû l'être, de toute dureté, de tout élément de résistance aux autres, de tout égoïsme ; fragile à force de délicatesse, elle semblait à tout moment prête à se briser, à expirer en un pur flot de larmes, puis l'ayant seule près de moi, vue sans le masque du visage, j'y remarquais, pour la première fois, les chagrins qui l'avaient fêlée au cours de la vie ${ }^{5}$.

5 La voix est parvenue à fleur de peau, sans le corps qui l'enveloppe. Le téléphone l'a affectée, il l'a déplacée hors de toute dureté, hors de toute minéralité, dont il ne lui demeure plus que le résidu de sa poussière : une brisure qui s'entend. Le téléphone a ôté à la voix ses résistances de pierre pour la redonner toute entière, neuve et pourtant exténuée, à son régime d'air : une brise d'air mouillée des larmes qui coulent en elle.

Le téléphone a touché cette voix familière et elle en est devenue sublimement touchante. C'est toute l'expérience de sentir qui se donne ici, en passant, dans cette voix de passage. Si sentir est bien, comme le décrit Erwin Straus ${ }^{6}$, l'épreuve non d'une connaissance, mais d'un toucher, d'un contact tel que la chose sentie est comme dans le mouvement fragile d'un souffle qui entre en nous et par lequel nous entrons en elle. Et un toucher qui absente le monde objectif autant que nous-mêmes et qui nous laisse l'un à l'autre à l'état de traces, d'atmosphères réciproques : très proches et très lointains. Il en est ainsi, de façon exemplaire pour Erwin Straus, des moments de crépuscule et de brouillard dans lesquels nous perdons notre chemin et prenons pleinement corps dans le pur sentir, dans l'épreuve du contact et de la distance des choses et de nous-mêmes. 
La voix de sa grand-mère est pour le narrateur comme un brouillard qui l'envahit, l'incorpore et qui, semblable à un courant d'air, rejoint le vide d'où elle a émané.

7 Elle est une voix première, jamais entendue, et une voix d'outre-tombe, déjà morte, accessible seulement à l'accueil réminiscent de l'écoute et qui toujours survivra, en un temps infiniment retrouvé, c'est-à-dire aussi perdu. Pour l'écrivain qu'est devenu le narrateur, nul doute que cette expérience du «souffle indistinct» dans la voix de sa grand-mère est un moment d'appel de l'Art. Comme le veut une longue tradition de la philosophie grecque depuis Platon, et comme la commente Jean-Louis Chrétien ${ }^{7}$, le beau, kalon, a sa source enchantée et lumineuse dans l'appeler, dans ce qui vient d'un appel : kalein. En commentant le Phèdre de Platon, Hermias parvient à cette formule si dense : «Cher en effet est le beau, étant ce qui appelle vers lui-même et convertit ${ }^{8}$ ». Le beau serait ainsi comme une aspiration. L'appel vient de ce qui se dégage en lui de supplément : un surcroît de don qui nous appelle, une ouverture qui nous dépasse, jaillie de l'invisible et de l'inaudible. La beauté a quelque chose à nous souffler. Et si l'on écoute bien, le téléphone, où le beau s'est chargé d'atmosphère, permet que l'on s'en approche. C'est une requête à l'oreille qui se délivre, parce qu'elle fait prendre conscience que rien de nous ni des choses ne suffit, qu'autre chose les porte, tel un vent portant.

8 Pour le narrateur de la Recherche, une voix réclame sa vocation: devenir écrivain (écrivain asthmatique) jusqu'à son dernier souffle; un écrivain qui ne cessera plus d'écouter d'où le monde appelle et d'ouvrir avec son œuvre le monde pour qu'il appelle. La voix entendue à Doncières, " ce jour-là », est de qualité maternelle et ancestrale, elle est d'arrière-mère. Elle se situe plus loin que la voix de la mère, elle qui lit à haute voix François le Champi au début de La Recherche; elle n'est pas une origine mais plutôt un milieu ouvert, le flux d'une musique sans partition, une trame légère, un paysage sans image. Son pneuma vient encore d'au-delà et de ce lointain en même temps si au bord, elle appelle l'écrivain. Elle lui fait sentir le beau, qui, dans le tissu natif de sa douceur et de sa fragilité, se fait entendre, ou plutôt appelle.

9 Le narrateur ne s'y attendait pas. Il a même cru qu'il n'aurait jamais sa grand-mère au téléphone, tant la ligne était encombrée de parasites. L'appel est donc arrivé à l'occasion de cette surprise, fulgurant comme un «miracle » écrit Proust. La voix est passée entre le temps et l'espace, conduite et diffusée par l'élan de l'éphémère, dans une intention d'être. Le téléphone a créé ce moment favorable. Sa puissance de flux a dégagé un intervalle, prenant le temps et l'espace au dépourvu, prenant aussi l'être qui l'a reçu au dépourvu et inventant une rencontre, beaucoup plus essentielle qu'une communication. Par cet intervalle, doux et fragile, dans cette fêlure de la voix au cœur de l'occasion, une traversée s'accomplit, intense, intranquille, vibrante, effusive. Elle est mélancolique aussi, puisque inséparable d'une perte, et elle est heureuse, apportant une vérité nouvelle, l'envie de l'œuvre résonnant de l'absence. "L'absence est, peutêtre, écrit Pierre Fédida, l'œuvre de l'art ${ }^{9}{ }^{\prime}$.

Rien ne serait évidemment possible sans l'effort de recevoir l'appel et le désir d'y répondre. L'artiste alors naît dans l'écoute, l'oreille et les yeux grand ouverts; il pénètre l'immanence du monde pour enregistrer toutes les sortes de souffles et les mener jusqu'à la forme transitoire, incertaine de la voix et de l'image. C'est d'une esthésique, une esthésique du milieu, qu'il s'agit, une sensibilité aiguë à l'atmosphère frissonnante d'un "temps-flux ${ }^{10}$ ", cerné de mort et ému de vie, sur les ondes de l'absence. 


\section{Genèse du poème, le souffle de la parole. La Fontaine}

" comme un peu d'air dans une main ouverte »

André du Bouchet

En 1678 déjà, La Fontaine écrit sur le téléphone; une fable qui raconte aussi la naissance du chant poétique. Un apologue invente le téléphone pour faire entendre le souffle d'un chant, remonté de la vague des morts :

« Le Rieur et les Poissons » (VIII, 8)

On cherche les Rieurs; et moi je les évite.

Cet art veut sur tout autre un suprême mérite.

Dieu ne créa que pour les sots

Les méchants diseurs de bons mots.

J'en vais peut-être en une Fable

Introduire un ; peut-être aussi

Que quelqu'un trouvera que j'aurai réussi.

Un Rieur était à la table

D'un Financier ; et n'avait en son coin

Que de petits poissons : tous les gros étaient loin.

Il prend donc les menus, puis leur parle à l'oreille,

Et puis il feint à la pareille,

D'écouter leur réponse. On demeura surpris :

Cela suspendit les esprits.

Le Rieur alors d'un ton sage

Dit qu'il craignait qu'un sien ami

Pour les grandes Indes parti,

N'eût depuis un an fait naufrage.

Il s'en informait donc à ce menu fretin :

Mais tous lui répondaient qu'ils n'étaient pas d'un âge

À savoir au vrai son destin ;

Les gros en sauraient davantage.

N'en puis-je donc, Messieurs, un gros interroger?

De dire si la compagnie

Prit goût à sa plaisanterie,

J'en doute ; mais enfin, il les sut engager

À lui servir d'un monstre assez vieux pour lui dire

Tous les noms des chercheurs de mondes inconnus

Qui n'en étaient pas revenus,

Et que depuis cent ans sous l'abîme avaient vus

Les anciens du vaste empire.

12 Au plan de table du financier, les assiettes sociales sont bien distribuées: les commensaux aisés (les gros poissons) occupent les bonnes positions, ils mangent copieusement; le rieur, lui, parasite du financier, est "en son coin ». Du menu, il ne récupère que le fretin et reste donc sur sa faim. À sa place, excentrique, l'information alimentaire est mal reçue, elle n'arrive à son bout de table qu'avec de la friture. Pour se faire mieux entendre, le rieur décide l'élaboration à l'improviste d'une plaisanterie destinée à ferrer les convives et pêcher au gros. Il saisit les petits poissons, leur parle à l'oreille ; après quoi, «à la pareille », comme le dit magiquement La Fontaine, il les porte à son oreille pour écouter leur réponse. L'appel fonctionne, la ligne est bonne : les poissons entendent bien, ils répondent. Pourtant ils sont morts et, on le sait de toute façon, même vivants ils sont opiniâtrement muets. Qu'importe, le rieur, un peu 
fabuliste en son genre, fait parler les poissons. Sa ventriloquie joueuse les ressuscite, les gonfle de souffle et les entend respirer. Les convives arrêtent de manger, leur bouche avide cesse tout bruit parasite : ils écoutent la voix d'eau des poissons. "Cela suspendit les esprits ». On le comprend aisément. Le rieur vient, dans sa main et dans sa parole, d'inventer le téléphone : pour passer sa commande et mieux manger. Il a trouvé dans l'appareil un prometteur instrument de revendication sociale, l'arme politique d'un pauvre, d'un affamé qui se rebiffe et veut en croquer.

Mais il se passe encore autre chose, de bien plus mystérieux et de bien plus ensorcelant. Quelque chose qui suspend vraiment les esprits : en inventant le téléphone, et même - il faut le reconnaitre - le portable, le rieur fait parler les morts ; avec son medium spirite, il provoque à cette table qu'il voudrait tournante, l'ébranlement d'une hantise.

Sur cette voie, la fable continue. Elle nous dit ce que se disent l'homme et les poissons : lui, cherche un ami disparu, eux sont trop jeunes pour en savoir quelque chose. Seuls les gros, plus vieux, pourraient faire remonter l'information. C'est à leur mémoire que le rieur en appelle, réclamant donc qu'on lui serve enfin une grosse et généreuse pièce.

Ici manger ne compte plus tout à fait ou alors cette table est celle d'un repas funèbre. On y parle des morts, de ceux qu'on a perdus de vue, dans l'abîme du temps, dans son gouffre de mer.

Le rieur appelle. Il a l'oreille collée à son poisson-conque. Il entend la mer et sa rumeur, ses vagues d'ondes, de la profondeur de son tombeau à sa surface. Le rieur appelle, devant les convives qui n'en reviennent pas, muets comme des carpes, il appelle ceux qui ne sont pas revenus des voyages, de la vie: il se donne le téléphone pour les rappeler. Les gros poissons en sont les formes survivantes. La Fontaine rêve des morts dans leur milieu d'eau, milieu grave, puisqu'il est brassé de naufrages; mais milieu suffisamment disponible, moins lourd que fluide, pour conduire - milieu conducteur donc -, pour conduire le ressac des morts. En riant, la bouche d'un rieur fait des vagues, des courants à drainer les poissons, à prendre au filet - de voix - les morts.

Dans leurs corps de poissons, les morts se tendent au contact. Ils étirent un intervalle d'air et fendent l'espace, comme le temps. Ils ont moulé l'air dans l'eau, moulé un mouvement de l'air dans l'eau. Quand l'un des élèves de François Chauveau, le premier illustrateur, le premier illustrateur des fables, veut montrer cette scène (ou cette cène), il place le rieur à gauche de sa vignette (fig. 1) : il le dessine écartant les mains pour suggérer la taille du poisson à lui faire parvenir, et à laquelle correspond la belle pièce à droite. Le rieur moule le poisson comme une forme du manque, et comme une forme de l'air, plutôt comme un milieu d'air, un interstice entre ses mains. De la bouche à ses mains faisant les parenthèses, tout le corps du rieur veut l'ouverture, ménageant ainsi un passage à la mort revenue. Revenue par l'écartement du temps et par celui de l'espace que les poissons ont rendu possibles et dont ils sont, de leurs corps plastiques emplis d'air, les ex-pressions. Encore a-t-il fallu l'appel du rieur, l'appel à l'ami, la faim de son souvenir, suscitée par une occasion essentielle, celle du manque. Le rieur s'est approprié cette occasion, ce kaïros fugace, il s'est adapté à elle, en homme de la métis, en homme de l'art hélé par l'absence. 
Figure 1

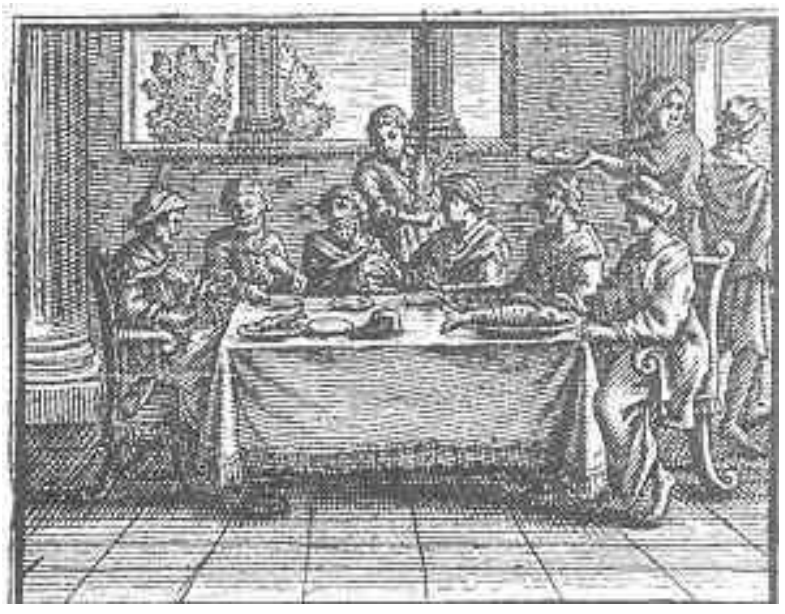

Atelier de François Chauveau, gravure pour «Le Rieur et les Poissons », Fables, VIII, 8, 1678.

Et ainsi vient un poète. Au terme de la fable, alors que le rieur récupère un vieux et gros poisson, La Fontaine prend la peine d'ajouter quatre vers pour contenir les paroles de l'animal. La ruse a réussi, et cependant, au-delà d'elle, flottent ces quatre vers inutiles, au haut bout du récit qui n'en avait pas besoin.

À dire vrai, on ne sait plus bien qui parle ici, dans ces vers en queue de poisson, dans ces vers magnifiques qui déploient le monde largement et avec largesse, comme un dernier don, le plus généreux. Qui parle ? Le rieur continue-t-il sa fable alors qu'il a de quoi manger ; excède-t-il alors son besoin par un simple désir de poète ? Le fabuliste at-il pris le relais? Est-ce le poisson lui-même dont nous entendrions étrangement la voix lyrique? C'est une voix qui parle, contenant toutes les voix possibles et advenant en plus. Elle est la somme de toutes les voix et leur reste. Une utopie. Cet au-delà de la voix, inassignable, sans origine, cette voix-esprit suspendue transmet les noms des morts, dont nous ne saurons jamais rien et justement pas les noms, pour toujours silencieux: "Tous les noms des chercheurs de mondes inconnus ». Les morts ont des visages, les vieux poissons les ont vus; ils ont des noms qu'ils ont transmis de leurs voix, sous l'eau. Ces visages et ces paroles sont devenus des " gestes d'air ", comme en parle Georges Didi-Huberman: des gestes d'air changés en poissons eux-mêmes métamorphosés en quelques vers ondoyants et furtifs. Dans leur va-et-vient (c'est l'étymologie de "vers", ce petit Fort-Da, dont ce poème des morts est fait), les morts disparaissent, apparaissent, réapparaissent fantomatiques et prestigieux, transfigurés, créateurs d'autres mondes, puisque fantômes : «chercheurs de mondes inconnus ». Les noms s'exhalent de la mer, et la voix dit même qu'ils viennent de "sous l'abîme ", où a habité le poisson géant qui parle. Le monstre a emporté la mer dans son corps; elle s'y est élastiquement resserrée, et l'animal en restitue la présence bruissante, peuplée des morts qui disent leurs noms. "Sous l'abîme", au-delà de l'infini, dans sa profondeur intarissable : de là, de cet arrière-monde, pour La Fontaine, remontent les noms.

Il les redonne dans un souffle, un souffle coupé et ce sont des vers pour un chant essoufflé. La Fontaine termine sa fable par quatre vers à couper le souffle, qu'il faut prononcer en haletant, la voix brisée, car le rythme prosodique de ce quatrain n'obéit qu'aux enjambements. Pas un vers donc qui ne suive l'autre sans l'insistance du souffle. La syntaxe y est tenue en l'air par les arrêts de la respiration, rythmée par la mort, 
douloureuse et endeuillée. Mais chaque moment de souffle s'y retrouve aussi comme une transition, et un lien, et une résurgence.

21 Le téléphone, échangeur d'appels et de réponses, milieu de souffles en allées et venues, donne vie au poème qui en redouble le pouvoir, dans son chant touchant. Il n'est pas question d'en juger : La Fontaine ne sait pas si les convives prirent goût à l'histoire du rieur, il ne sait pas non plus s'il a lui-même fait une bonne fable : "peut-être aussi / Quelqu'un trouvera que j'aurai réussi ». Ce qui ne peut être connu dans cette fable est à sentir, à éprouver. Rien d'autre ne l'intéresse et ne l'inspire.

\section{Genèse de l'image : le souffle du visage. Ernest Pignon-Ernest/James Turrell}

"Quand je ne vois rien, je vois l'air » André du Bouchet

En 1997, Ernest-Pignon Ernest installe dans des cabines téléphoniques un ensemble sérigraphique, environ 450 images, à Lyon et à Paris. Ce sont des êtres de papier, collés aux parois vitrées: des hommes et des femmes plaqués contre la transparence, simplifiés en pellicules, rayés de lignes noires et de segments blancs. Ernest PignonErnest laisse ces chairs plates dans les espaces publics des cabines pour un séjour éphémère, une transhumance de quelques jours. On a vu une femme nue, une autre criant, un homme brisé, appuyé contre la vitre, ou recroquevillé (fig. 2) ${ }^{11}$.

Figure 2

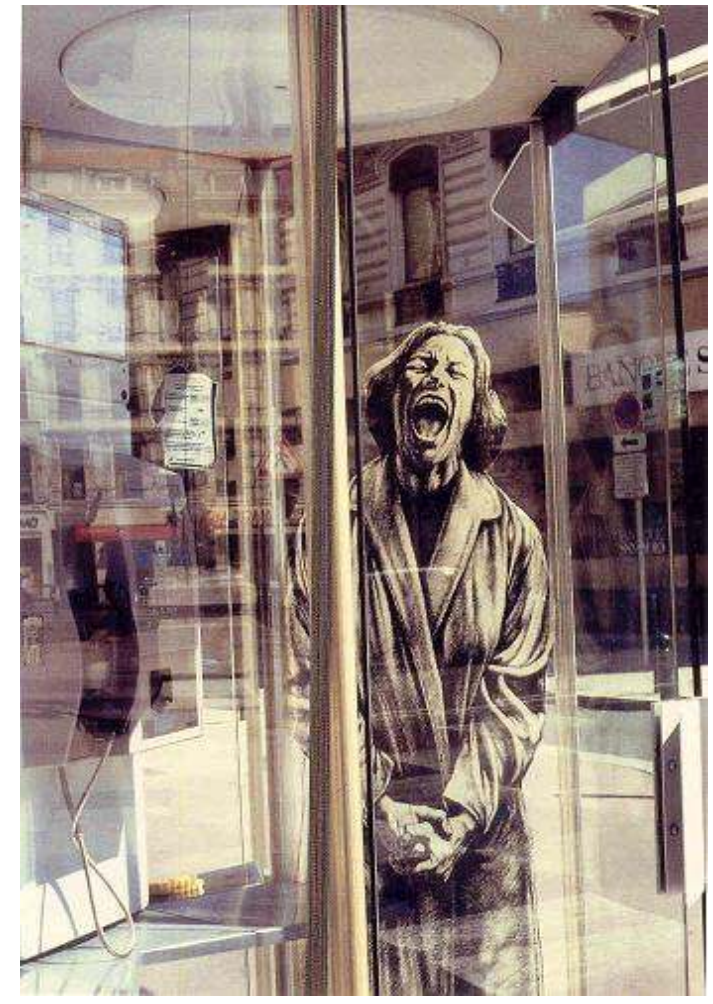

Ernest Pignon-Ernest, Cabines téléphoniques, installation, Lyon, 1997 

et de l'abandon. Chacun d'entre eux endosse son histoire dans les sillons des traits; ce sont eux qui leur ont donné leurs ombres de corps (ou leurs corps d'ombre). Chaque histoire est inconnue. Il n'en subsiste que des bouches ouvertes, des figures déchirées de douleur, la chair abattue, une humanité appelante. l'effort vers la voix mais vers le visage. Le souffle des morts ne s'est pas vaporisé dans le rythme d'un chant, issu de l'inaudible : il a pris corps dans la condensation frêle d'une figure, sortie de l'invisible. Pourtant, de La Fontaine à Ernest Pignon-Ernest, les expériences esthétiques se croisent ou s'appellent: dans la fable, il fallait entendre pour voir (les anciens du vaste monde avait vu les voyageurs naufragés et ils faisaient alors entendre, à l'oreille, leurs visages); dans la rue, il faut voir pour entendre, il faut que l'œil écoute. Mais nous sommes concernés par le même appel: l'appel des morts par leur beauté, qu'elle se donne la chance de l'image ou du poème.

Grâce à Ernest Pignon-Ernest, le téléphone, dans le ventre de ses cabines, dans leur petit antre, fait pousser une série de voix silencieusement parvenues jusqu'au dessin des images. La transparence agit comme un milieu, prolongeant et développant le téléphone lui-même, qui a agité les ondes de l'appel et leur a accordé l'énergie d'apparaître. Du souffle a passé, par la bouche du combiné, par son chas d'aiguille ; il s'est diffusé, il s'est répandu, il se meut dans l'aquarium. Les formes qui surviennent peuvent flotter, autant que remonter ou choir : une sorte d'apesanteur leur offre tout leur poids pathétique de gravité. La nuit, les lampes au plafond des cabines font tomber un halo vertical et provoquent de nouvelles modifications. Les reflets des néons extérieurs, et puis les murs, les ombres, les éclats de lumière et de couleur, des bouts d'immeubles et de rues, des silhouettes de passants décomposées, la nuit et le jour, ajoutent leurs empreintes locales, diffractées et absorbées dans le plan unique, mobile de la transparence. Les figures subissent les métamorphoses de deux atmosphères qui se rencontrent en milieu transparent: l'atmosphère des morts insinuée par le téléphone et répandue dans la cabine et l'atmosphère de la ville, éclatée en gouttes de couleurs et de formes, en souffles partiels de lumière et en minces opacités. La cabine transparente, peut-être même diaphane, fonds et surface mêlés, arêtes et volume confondus, réalise ou précipite l'entrelacs d'une intériorité et d'une extériorité qui se touchent et activent une moire cristalline. Cette rencontre n'oublie pas la séparation et l'absence : la transparente œuvre à la fois comme distance et comme coalescence.

Chaque figure d'homme et de femme aimante cette rencontre. Les corps donnent l'impression de rassembler les rumeurs diffusées par le combiné téléphonique, sans même qu'il soit décroché, et les rumeurs aussi qui foisonnent sur la paroi du verre ; et en même temps, ils semblent se ramifier dans leur environnement, se diffuser dans le verre et ses mosaïques visuelles. Il est même possible que la cabine soit la création de ces silhouettes graphiques, quelque chose comme leurs larmes condensées, ou leurs buées. Un homme, par exemple, aux oreilles bouchées, depuis le fond d'ombre de sa bouche, s'enveloppe de souffle, il s'habille dans son vêtement vitré en même temps qu'il l'aspire à lui. Les empreintes éphémères, à peine tatouées sur la transparence, sont « dynamophores » (elles conduisent le souffle comme le souffle les conduit) et elles sont «dynamomorphes »: elles ne font que passer, sous de multiples formes (le temps les leur souffle). 

d'Ernest Pignon-Ernest, par la liquidité de l'encre. L'encre, variablement dense, s'approprie un premier milieu, celui du papier. Elle le soumet à des modulations d'intensités, de liquéfactions, des bavures et des taches et elle l'oblige aussi à des réactions (elle-même devenant milieu). Le pinceau tourne, glisse, il n'accroche pas complètement, il mêle le jour et la nuit, les fait circuler l'un dans l'autre, il les frotte, il les tresse. souffle plus insistant : les traits s'affirment, davantage crayonnés, le dessin se raidit sur le papier et peut se dresser. S'effectue alors le transfert dans un autre milieu plus vaste, plus riche de virtualités encore: les dessins fleurissent les cabines, leurs milieux de mort et de vie aussi, de métamorphoses et d'intensités. Dans les cabines, la présence entre-absente des images s'agrandit ; elle se donne un moment en partage, le temps que dure son existence à l'air libre.

Nous pouvons même entrer dans l'image, si nous le désirons (fig. 3).

Figure 3

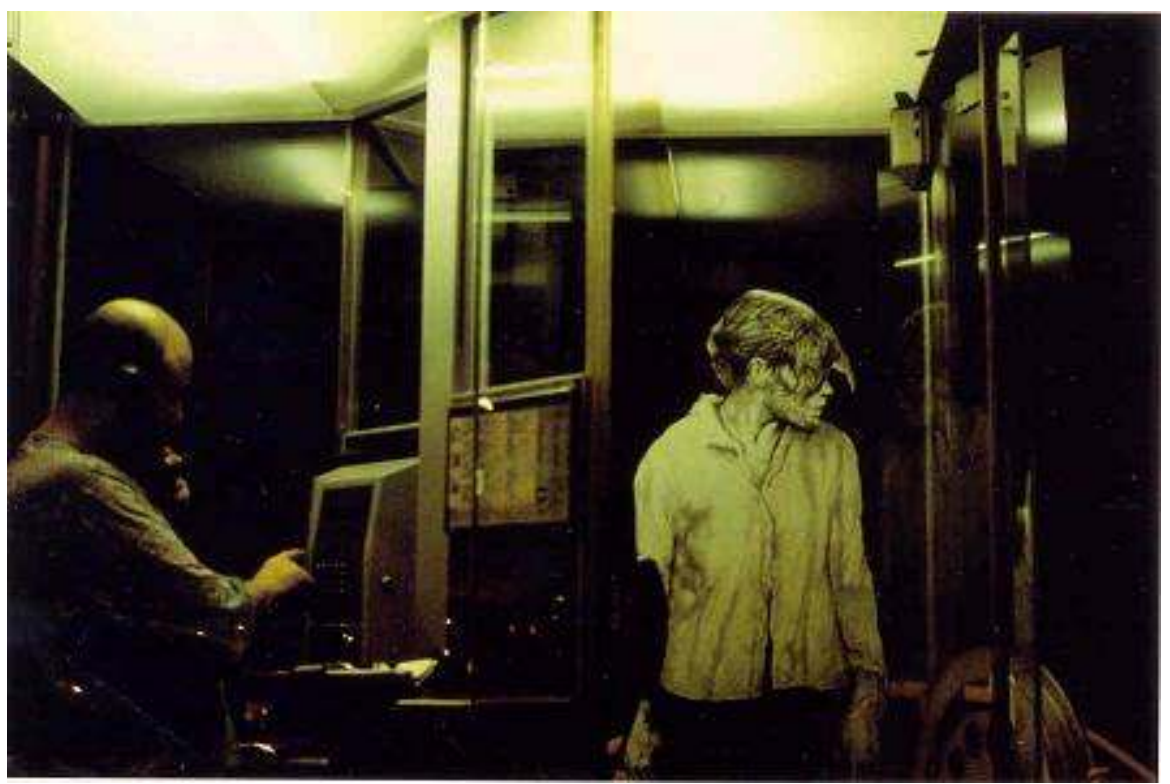

Ernest Pignon-Ernest, Cabines téléphoniques, installation, Lyon, 1997

La transparence nous y invite. Nous sommes invités à téléphoner, à appeler nousmêmes ou à répondre à un appel. Et alors l'expérience serait extrême, extrêmement touchante, de téléphoner ainsi à nos morts, de les voir un peu sous des visages et des corps inconnus, par là universels, comme des porte-morts. Et c'est aussi comme si nous téléphonions à notre propre mort. Nous pouvons la sentir, tout autour de nous, nous prendre, au bord de notre asphyxie, au bord de l'hallucination. Nous sommes dans la sépulture des morts, dans le tombeau né de leur appel, un temps libéré.

La verticalité acquise par les images dans les cabines, leur exposition à l'air et à la lumière est toute provisoire (leur accrochage est inséparable d'un décrochage, comme pour le combiné du téléphone dans les cabines) : le noir et le blanc des images, ombres et hiatus, ensemble déjà fabriquent la perte et pour ainsi dire le suicide de l'image (c'est 
ici la dialectique du milieu) : ils la creusent et l'obscurcissent comme ils l'amènent un laps de temps au visible.

Ernest Pignon-Ernest abandonne chaque image au temps; chaque image est vouée à se déchirer, à se fractionner, à s'estomper, à s'effondrer et se défaire, comme un linge détaché, "un haillon cou[lant] au fond de l'eau» (V. Hugo). Le temps passant, trépassant dans l'espace rappelle les images à leur mortalité, à leur devenir-souffle. La colle n'est qu'une fixité transitoire, et elle-même déjà mange l'image. Le souffle ne cesse de travailler le papier et le dessin, et de les remettre à leur destin de poussière, de vide et de vent. Les images sont déjà en habit de deuil, elles ont pris son clair-obscur, le blanc et noir de la souffrance qui accentue toujours les contrastes. Elles prennent corps par un mélange de cendres et de silence, de visage et de voix soufflés (comme on pourrait le dire à la suite d'une explosion). Les figures, dans leur milieu, possèdent ainsi la double qualité, contradictoire, du souffle : la lenteur et l'explosivité. Ce sont autant des qualités de temps que des qualités d'espace, créant en commun l'atmosphère des choses : lenteur du souffle qui s'accorde le temps de faire un espace, de l'envoûter, d'en prendre possession et d'en faire un lieu de hantise ; explosivité qui concentre, façonne un volume, une demeure et en même temps qui oblige ce volume à l'indétermination et à la disparition.

C'est cette même lenteur et cette même explosivité du souffle vers l'image que le plasticien américain James Turrell pousse jusqu'à éblouissement de la couleur, dans un dispositif de cabines téléphoniques créé au cours des années 90 (Telephone booth) (fig. 4).

Figure 4

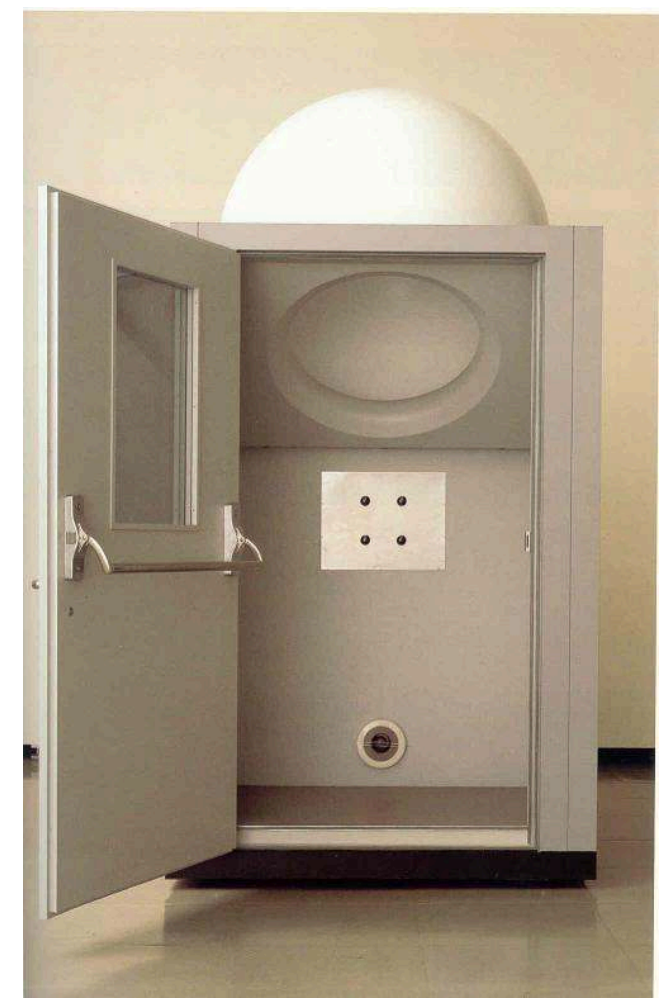

James Turrell, Call waiting, 1997

James Turrell y rejette la transparence, il veut une étanchéité absolue du milieu sensible, un repli sur le monde du dedans et une immersion. L'événement qui survient 
dans ses cabines associe la double traversée de la couleur et du son. Le spectateur peut lui-même, une fois installé, régler les influx lumineux. La lumière actualise alors progressivement et variablement son irradiation, dans une sorte de casque ou de petite voûte céleste fermée, où doit se loger la tête de celui qui est à l'intérieur. Pendant la levée de la lumière, un bruit de souffle ou de vent lui-même levé s'entend dans la cabine et l'emplit ${ }^{12}$. Le lieu, de manière coïncidente, scintille de couleur et de vent. Aucune voix n'est décelable, aucun visage ne prend forme, fût-ce passagèrement comme dans la série d'Ernest Pignon-Ernest. Ou alors puisque le vent souffle sa couleur sur le visage, l'impression peut être celle d'un face à face : la couleur aurait un visage, elle serait envisagée aux dimensions du rond de lumière ; avec pour l'ovale de ce visage aussi les dimensions d'une bouche (fig. 5).

Figure 5


James Turrell, Call waiting, 1997

Ce serait, si l'on veut, être dans le regard cyclopéen d'une bouche qui souffle de la couleur, un regard cracheur de feux. Un regard qui fait un $\mathrm{O}$ de surprise et de cri, en gros plan flamboyant.

La couleur n'est donc pas frontale, elle n'est pas fixée. Elle repousse les limites de sa mandorle, elle l'effrange. Se comportant comme une texture enveloppante, happante, elle baigne, elle teinte le visage et le corps du spectateur qui est baptisé de lumière. Autrement dit, elle emporte la forme, elle l'appelle de sa voix de sirène, elle l'éclaire et la sort d'elle-même, elle lui enlève sa distinction et sa netteté au profit de la puissance d'incandescence des bords. L'absence déborde, elle nous déborde en une sorte d'extase énergétique, la tête dans la couleur, dans un air brillant de tous ses feux.

Cette expérience, nous savons qu'elle est proche de celle que nous pouvons avoir quand nous fermons très forts les yeux. Dans cette nuit de nos paupières, à l'intérieur de notre regard, comme sous lui, surgit la même éclaboussure stellaire que dans les cabines 
téléphoniques à ciel fermé de James Turrell. Nous pouvons ainsi appeler la couleur, avec le regard du rêveur ou celui du mort, les "yeux éteints", selon le fragment d'Héraclite. C'est la couleur indécise des yeux de la mort qui souffle dans nos yeux retournés et que nous habitons alors : comme regarder dans un sépulcre coloré ouvert au vent. Voilà ce que reproduit James Turrell dans sa cabine : il fait un lieu de cette expérience du dedans de notre regard, un lieu où l'absence peut nous habiter et où nous pouvons l'habiter.

Dans les cabines d'Ernest Pignon-Ernest, nous étions encore, d'une manière ou d'une autre, dans notre milieu, nous pouvions continuer à téléphoner. Les téléphones marchaient vraiment. Nous pouvions même, à la rigueur, ne pas voir les personnages de papier, il nous était possible de les ignorer avec une cruelle indifférence. Quant aux fantômes, ils avaient repris notre forme pour nous visiter, ils nous ressemblaient, dans cette transparence-miroir. James Turrell, lui, dispose ailleurs ses cabines, il les sort de leur milieu habituel, il les délocalise pour créer un autre milieu, simplifié à la couleur soufflée ou au souffle coloré : «La perception, écrit James Turrell, n'est informée que par un événement qui est en train de se produire ${ }^{13}$ ». Il en résulte une rencontre plus mystérieuse encore, qui invente la zone frontière, labile où nous venons aux morts et où les morts viennent à nous: un milieu intermédiaire, de pur échange. Maurice Merleau-Ponty a une magnifique et ample expression pour nommer la sensation que nous pouvons avoir lorsque "nous nous laissons être au monde sans l'assumer activement, ou dans les maladies qui favorisent cette attitude ${ }^{14} »$ : dans des sortes d'état second, d'épuisement, de transes passagères. Dans ces conditions, dit Merleau-Ponty, "les couleurs ne se condensent plus en couleurs superficielles, elles diffusent autour des objets et deviennent couleurs atmosphériques"; c'est là ce qu'il appelle la "voluminosité ", «simple ouverture de la perception à un fantôme de chose à peine qualifié ${ }^{15} »$.

Comme les astres ont leur dazzling où, à leur pourtour, tremble une clarté qui captive et presque aveugle, les cabines de James Turrell nous attirent dans le vertige de la perception qui absorbe notre rétine et autour d'elle tout notre corps. Souffle et couleur, couleur du volume, volume de la couleur, l'onde plastique du téléphone nous métamorphose en lieu coloré. Elle nous touche comme Midas changeait les corps en or. Nous baignons dans le pactole de l'appel, sans découpe, sans profondeur, sans surface et finalement sans forme. Neutralisés dans l'emphase de la lumière homogène.

À partir du téléphone, investi au plus loin et au plus proche de nous comme medium spirite, Proust, La Fontaine, Pignon-Ernest, Turrell ont chacun imaginé des expériences de voluminosité. Elles leur ont fait rêver à la naissance des poèmes et à celle des images, comme accueil, c'est-à-dire réponse à l'appel obsédant du « souffle indistinct » de l'absence, à « l'absence qui me tient lieu de souffle » (André du Bouchet).

BIBLIOGRAPHIE

Buci-Glucksmann Christine, Esthétique de l'éphémère, Paris, Galilée, 2003. 
Chrétien Jean-Louis, L'appel et la réponse, Paris, Minuit, 1992.

Fédida Pierre, L’Absence, Paris, Gallimard, 1978.

Fédida Pierre, Le Site de l'étranger. La situation psychanalytique, Paris, PUF, 1995.

Fédida Pierre, «L'Ombre du reflet. L'émanation des ancêtres », in La Part de l'œil, n 19, 2003-2004.

Hermias, In Platonis Phaedrum Scholia, Paris, P. Couvreur, 1901.

Leplatre Olivier, Appels à communication. Écritures du téléphone, Paris, L'Harmattan, 2005.

Merleau-Ponty Maurice, Phénoménologie de la perception, Paris, Gallimard, 1945.

Meuris Jacques, James Turrell. La perception est le médium, Bruxelles, Éditions de La Lettre volée, 1995.

Proust Marcel, Du côté de Guermantes, in À la recherche du temps perdu, Paris, NRF-Gallimard (La Pléiade), t. II, 1998.

Straus Erwin, Du sens des sens. Contribution à l'étude des fondements de la psychologie, Grenoble, Jérôme Million, 1989.

Tellenbach Hubertus, Goût et atmosphère, Paris, PUF, 1983.

\section{NOTES}

1. H. Tellenbach, Goût et atmosphère, Paris, PUF, 1983.

2. P. Fédida, Le Site de l'étranger. La situation psychanalytique, Paris, PUF, 1995, p. 187-220.

3. P. Fédida, «L'Ombre du reflet. L'émanation des ancêtres », in La Part de l'œil, nº 19, 2003-2004, p. 195.

4. Sur l'élégiaque téléphonique, je me permets de renvoyer à mon livre : Appels à communication. Écritures du téléphone, Paris, L'Harmattan, 2005.

5. M. Proust, Du côté de Guermantes, in À la recherche du temps perdu, Paris, NRF-Gallimard (La Pléiade), 1998, t. II, p. 432-433.

6. E. Straus, Du sens des sens. Contribution à l'étude des fondements de la psychologie, Grenoble, Jérôme Million, 1989.

7. J.-L. Chrétien, L'appel et la réponse, Paris, Minuit, 1992, p. 22-27.

8. Hermias, In Platonis Phaedrum Scholia, Paris, P.Couvreur, 1901, p. 13, cité par J.-L.Chrétien p. $18-10$.

9. P. Fédida, L'Absence, Paris, Gallimard, 1978, p. 7.

10. Ch. Buci-Glucksmann, Esthétique de l'éphémère, Paris, Galilée, 2003.

11. Voir le site personnel d'Ernest Pignon-Ernest : http://www.pignon-ernest.com/

12. On peut trouver une animation du Telephone booth sur le site: http://www.kunst.unistuttgart.de/seminar/turrell_klein

13. Entretien avec Julia Brown Turrell, dans Galeries Magazine, Paris, 1991. Voir J. Meuris, James Turrell. La perception est le médium, Bruxelles, Éditions de La Lettre volée, 1995.

14. M. Merleau-Ponty, Phénoménologie de la perception, Paris, Gallimard, 1945, p. 307-308.

15. Ibid. 


\section{RÉSUMÉS}

Dans son milieu d'espace et de temps, dans son milieu d'air, le téléphone a vocation à être l'instrument sensible de l'appel des morts. À partir du téléphone, appréhendé comme medium spirite, Proust, La Fontaine, Ernest Pignon-Ernest, James Turrell ont chacun imaginé des expériences de voluminosité. Elles leur ont fait rêver à la naissance des poèmes et à celle des images, comme accueil, c'est-à-dire comme réponse au "souffle indistinct» (P. Fédida) de l'absence.

\section{AUTEUR}

\section{OLIVIER LEPLATRE}

Professeur en littérature, université Jean Moulin Lyon 3 